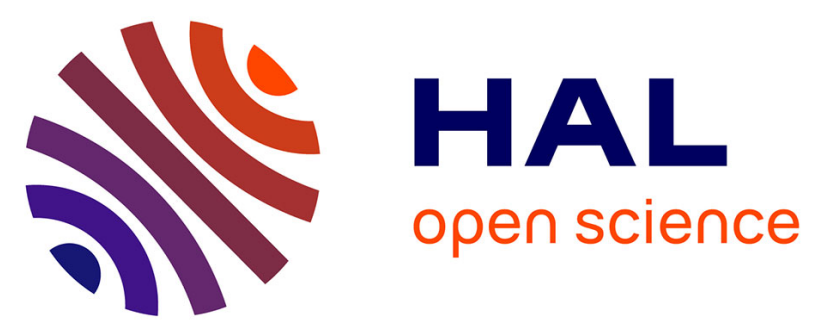

\title{
Design and Operationalization of Connectivist Activities: an Approach through Business Process Management
}

\author{
Aicha Bakki, Lahcen Oubahssi, Sébastien George
}

\section{To cite this version:}

Aicha Bakki, Lahcen Oubahssi, Sébastien George. Design and Operationalization of Connectivist Activities: an Approach through Business Process Management. The 14th European Conference on Technology Enhanced Learning, Sep 2019, Delft, Netherlands. pp.251-265, 10.1007/978-3-030-297367_19. hal-02170576

\section{HAL Id: hal-02170576 \\ https://hal-univ-lemans.archives-ouvertes.fr/hal-02170576}

Submitted on 26 Sep 2019

HAL is a multi-disciplinary open access archive for the deposit and dissemination of scientific research documents, whether they are published or not. The documents may come from teaching and research institutions in France or abroad, or from public or private research centers.
L'archive ouverte pluridisciplinaire HAL, est destinée au dépôt et à la diffusion de documents scientifiques de niveau recherche, publiés ou non, émanant des établissements d'enseignement et de recherche français ou étrangers, des laboratoires publics ou privés. 


\title{
Design and Operationalization of Connectivist Activities: an Approach through Business Process Management
}

\author{
Aïcha Bakki ${ }^{1,2}$, Lahcen Oubahssi ${ }^{1}$, Sébastien George ${ }^{1}$ \\ ${ }^{1}$ Le Mans Université, LIUM, EA 4023, Laboratoire d'Informatique de l'Université de Mans, \\ Avenue Messiaen, 72085 LE MANS CEDEX 9, France. \\ ${ }^{2}$ Ibn Zohr University, IRF-SIC Laboratory, Agadir, Morocco.
}

\begin{abstract}
The work presented in this paper focuses on massive open online course (MOOC) environments, and more specifically on the activity of designing and implementing pedagogical scenarios for a connectivist MOOC (cMOOC). This paper presents a research work, which aims to propose a model and tool to support the design of connectivist MOOC scenarios. The major contribution of this work is a visual authoring tool that is intended for the design and deployment of cMOOC-oriented scenarios. The tool is based on the BPMN notation that we have extended to suit our objectives. The tool was evaluated primarily from the point of view of utility and usability. The findings confirm that the tool can be used to design connectivist pedagogical scenarios and can provide all the necessary elements to operationalize such courses.
\end{abstract}

Keywords: TEL, MOOC, cMOOC, authoring tool, BPMN, connectivism.

\section{Introduction: Motivation and Aims}

The research work presented in this paper is part of a general issue of TEL. It deals, more specifically, with pedagogical scenario design of connectivist MOOCs. Nowadays, MOOCs correspond to an effective learning method, which offers a free, distributed, and open access to education and training. They have increased remarkably by adopting collaborative mechanisms and offering new features promoting communication and exchange between learners. Between new trends and innovative pedagogical concepts, MOOCs have stood out and have received acclaims, as well as criticisms on several levels. This research work has explored some of the multiple facets of MOOC as a research object through pedagogical design support and assistance to teachers. The intended purpose is to conceive models and implement tools to assist teachers in the cMOOC design process by taking into account complementary and plural aspects of elearning, through individual, collaborative, social and massive dimensions. With the advent of MOOCs in higher education, the stakeholders intended to transcribe the aspects of transmissive pedagogy into MOOCs: xMOOCs then appeared. Although this categorization is considered minimalist, we have relied on the distinction between $\mathrm{xMOOC}$ and $\mathrm{cMOOC}$ in our proposal. By comparing these types of MOOCs, some differences were observed, particularly in regards to: the roles played by the teacher 
and the learner, the pedagogical aspects targeted and the openness and freedom granted to learners [5]. Despite the potential benefits of cMOOCs, the literature review has shown that the most widely deployed types of MOOCs are xMOOCs. Based on an analysis of a panorama of 76 MOOCs, [33] revealed that only $10 \%$ of these courses can be categorized as cMOOCs. This observation was addressed by [24] who explained this by pointing out that the majority of teachers do not feel confident and are lacking the technical skills to deal with connectivist environments that are mainly focused on the use of technology. We share this point of view, and believe that a limitation for the emergence of cMOOCs is the lack of methodologies, models and tools to support pedagogical scenario development [3] as the current literature provides a description of pedagogical practices in cMOOCs in a purely descriptive manner. Based on this observation, we have hypothesized that modeling a cMOOC scenario and reifying it in an information system that is easy to use by teachers with no computer expertise will help them to move toward this type of MOOC. Our objective is to assist the teacher-designer who desires to create a cMOOC to produce a pedagogical scenario that respects the specificity of this kind of learning environment. From a conceptual point of view, the major difficulties faced by teachers are, firstly, how to put in place a scenario that emphasizes the new roles played by the learner, and secondly, the lack of adequate tools and/or models for designing cMOOC scenarios without prior knowledge of the underlying pedagogical model. Indeed, in order to meet teachers' expectations, an idea is therefore to use the benefits of Business Process Model and Notation (BPMN). More especially as the latter has been able to stand out in the TEL field by its graphic notation easily understandable by different actors as it provides a set of generic business process elements, independently from a specific domain; its compliance with the standard and as it is intended for different audiences and especially for computer novice users [14].

The remainder of the paper is structured as follow: in section 2, the cMOOC pedagogical scenario domain specifications are summarized. In section 3, we present overview of the BPMN and its benefits for connectivist context. In section 4, we present a brief description of the deployed extension design and method. Since the objective represents an artifact including extended notation and its technical implementation, section 5 present the proposed authoring tool. The section 6 highlights our proposal for the operationalization and deployment phase. The section 7 presents an evaluation of the proposed tool. The paper ends with a conclusion and an outlook on obtained results.

\section{Characteristic of a cMOOC Pedagogical Scenario}

cMOOCs designate connectivist MOOCs driven by the principles of pedagogical innovation in a widely interconnected social learning mode. They are based on "a sophisticated and innovative design" of learning practices [12], and involves the promotion of learning through collaboration, production, sharing and connections between peers in quasi-total openness. The openness of cMOOCs might suggest that pedagogical scenario building is not essential, and that this would be contrary to the underlying principles of a connectivist course. However, we believe that connectivist course design can be enhanced by the implementation of scenario-building practices. Indeed, despite 
this openness, it is essential to create suitable conditions for the emergence of connectivist activities. In this sense, several solutions have been suggested to facilitate the implementation of such courses [2,28]. These studies focus on cMOOCs from a theoretical point of view, and aim to demonstrate the value of a methodology for the scenario development and implementation of connectivist courses. They also offer frameworks that describe the main axes of the design process and the implementation of a cMOOC course, but define the administrative aspects rather than the elements that should be contained by a cMOOC scenario $[18,26,28]$. These studies confirm that cMOOCs rely on a specific conceptual model to help teachers to conceive such courses, but they didn't provide neither a model to describe a cMOOC pedagogical scenario nor concrete and simple software tools to design and deploy connectivist MOOCs.

One of the major difficulties faced by teachers wanting to design such courses is to determine how to model educational activities within this connectivist context. The issue lies in creating pedagogical and monitoring scenarios to support learners so that they do not feel overwhelmed. Designing such scenarios is challenging, since it requires an effective collaboration between the teacher and learners throughout the course. Another difficulty involves setting up cMOOCs in order to respects the freedom of learners to define their own educational objectives. In this perspective, teachers should not establish a specific linear course plan, but should suggest resources and activities that can guide learners toward the main objective of the $\mathrm{cMOOC}$ and then encourage them to create, produce and collaborate. These complexities require some mechanisms and methods that can guide and support teachers in the design of the cMOOC.

Pedagogical questions have been raised and constitute one of the major criteria to characterize cMOOCs. In this sense, a study of the pedagogical practices of a connectivist course is essential to define the elements that regulate a cMOOC scenario and model them. That being said, we had to conduct a literature review on the pedagogical aspects of cMOOCs. In accordance with the theoretical grounding of the connectivist approach, we found that cMOOCs are structured into four essential activities [16, 21]: Aggregating activities aim to encourage learners to read and consult the content and resources that are most relevant to their learning objectives. The learners are encouraged to read, choose and filter what is most relevant and appropriate to achieve their personal learning objectives. Remixing activities can be defined as interpreting the information collected during the aggregation phase and searching for relevant additional resources. Repurposing activities aim to support learners through an individual or group production process. Feed forwarding activities aim to encourage learners to share their products over the web. These are essentially transmission activities. We assume that a cMOOC scenario should contain all four groups of activities presented above. In the remainder of this paper, we will explain how we purposed to conceptualize this theoretical fundament through a technology-aware framework.

\section{BPMN as Pedagogical Language in cMOOC Context}

Before the advent of Learning Design (LD) tools, teachers used to create their scenarios using a narrative textual format. Such scenarios do not use a standardized template, 
meaning that it is difficult to disseminate and reuse them [20], and thus the IMS-LD specification emerged [13]. IMS-LD is essentially a description language that allows to model the lesson plan and describes roles and activities without handling the implementation processes [14]. Since IMS-LD is not an easy-to-understand process for the teachers [2, 24], the first generation of LD authoring tools was proposed.

A significant number of research works has proposed tools and languages to help with the visualization of learning designs, and these works can be divided into two main groups. The first concerns solutions that provide specific notations for the creation of a pedagogical scenario, but that are not implemented in a tool. The second group concerns visual modeling tools that offer teachers more abstract languages; these are visual or graphical tools, and their use is more intuitive [7]. Thus far, none of these tools have allowed teachers to design cMOOC and to automatically deploy them on a platform. In addition, some of these LD tools are directed toward a particular pedagogical approach, are specific to a particular platform, or meets the requirement of the IMS-LD standard which does not correspond to our needs because although it is a so-called pedagogically neutral standard, it has shown its limits for designing collaborative and constructivist situations $[10,17]$. Other works were inspired by business modeling, and specifically by the workflow approach. In this sense, BPMN is offered as an alternative to LD languages. Several studies have been conducted $[2,14]$ to illustrate and support the use of this workflow language within the LD process. BPMN has stood out in the educational field due to its advantages and in particular its expressiveness, its simplicity of use and the graphical representation of pedagogical scenarios.

The LD tools have advantages and disadvantages that influence their use and execution. Several studies have been carried out to specify the requirements and/or needs for LD tools and languages $[8,25]$. According to these works, from a techno-descriptive point of view, BPMN has several advantages that offer a teacher an intuitive tool, through its visual notation, its formal character and its level of stratification in layers that offers a different representation for each modeling element. From a pedagogical point of view, BPMN allows the representation of learning modalities by specifying the different activities, their dependencies and especially by offering the possibility to define a non-linear pedagogical scenario with several connections.

According to [17], when a learning environment model is confronted with significant variations in its initial conditions, the adaptation of the model that supposedly represents it becomes very difficult: this is the case of connectivism approach. Hence, to successfully cope with the complexity of the cMOOC learning process and its dynamics the targeted LD systems have to facilitate the cMOOC scenario design process in its entirety. Such support must include tools that provide a support for all components of the process, as well as possibilities to simply manage changes in that process. Based on all the points above, one possible approach to provide such a support might be a reuse of the experiences, and practices from business processes. Reusing the BPM notation and extending it with domain-specific concepts are expected to be less expensive than deploying a domain specific modeling language from scratch. However, in order to meet our objective to offer the teacher support in the design of cMOOC-oriented pedagogical scenarios, the use of BPMN is not directly conceivable. Since BPM notations are meta-modeling notations, a pedagogy-specific vocabulary based on these abstract elements should be constructed [19]. In this sense, there are research developments that 
need to be realized for a successful application of BPMN in our context. Those developments aim to support the whole cycle of a cMOOC pedagogical scenario, i.e., first, facilities for conceiving cMOOC scenarios are addressed; and second, mechanisms for automatic mapping, deploying, and executing of pedagogical scenario within the available Learning Management Systems (LMS) are taken into account. To do so, we have to first propose an extension of the BPMN concept to take into account the specificities of a cMOOC-oriented pedagogical scenario (section 4). Then, we embed the extended meta-model and notation in an authoring tool (sections 5 and 6). Finally, we develop a mapping and automatic-deployment service to existing LMS (section 7).

\section{$4 \quad$ BPM Notation and Meta-model Extension}

In 2011, OMG introduced the latest version of BPMN: BPMN 2.0. The BPMN 2.0 specifications define the different graphical notations that form the basic set of BPMN elements. It is one of very few modeling languages that provides generic extension elements within the meta-model that enables the definition of domain-specific language extensions [27]. Nevertheless, BPMN does not provide any methodological guidance or support to comply with domain-specific extension issues. In this sense, [31] proposed a Method for the Development of BPMN Extensions that consists of the steps listed below: (1) Definition of a Conceptual Domain Model of the Extension (CDME) describing the concepts of the domain to be represented in extended BPMN models and their relationships with the concepts of the BPMN meta-model. (2) Definition of a BPMN plus Extensions model (BPMN+X) describing an extension based on the specification of the BPMN extension mechanism. (3) Transformation of the BPMN+X model into an XML Schema Extension Definition Model. (4) Transformation of the XML Schema Extension Model into an XML Schema Extension Definition Document.

[9] proposed an extension of [31] method by integrating the analysis of the domain and its conceptualization. The authors proclaim that for a domain-specific extension, a domain requirement analysis is important in order to explicit all the necessary concepts of the domain and its semantics, and to consider whether the domain-specific concept is semantically equivalent to an existing BPMN element or not. As our aim is to propose a cMOOC pedagogical scenario specific extension, we consider as necessary to integrate the equivalence check procedure proposed by the authors. For this purpose, we are illustrating essentially how the first two steps of the [31] procedure model will be applied. We also add an Equivalence Mapping according to Equivalence check procedure proposed by [9]. Referring to the presented process model (Fig. 1.) the design of the proposed extension is briefly presented below.

Domain analysis. In order to conceptualize the targeted educational domain, we have analyzed the pedagogical concepts related to the connectivist approach, and we propose a model, named cORPS [4], that allows expressing the structural properties specific to a connectivist environment as well as the temporal properties.

Equivalence Mapping. The BPMN extension is based on specific domain concepts of our proposed Model. Each of these concepts are semantically compared to the BPMN concepts in order to define the needed extension in form of a new element or properties. 
As result of equivalence mapping a classification of the connectivist element as BPMN element or as an extension concept is made. The first one refers to the elements of our model that has an equivalent BMPN concept and second one corresponds to the elements who have no equivalent or who had no obvious semantic matching with standard elements, but rather situational discussion is necessary in order to provide arguments for a possible mapping.

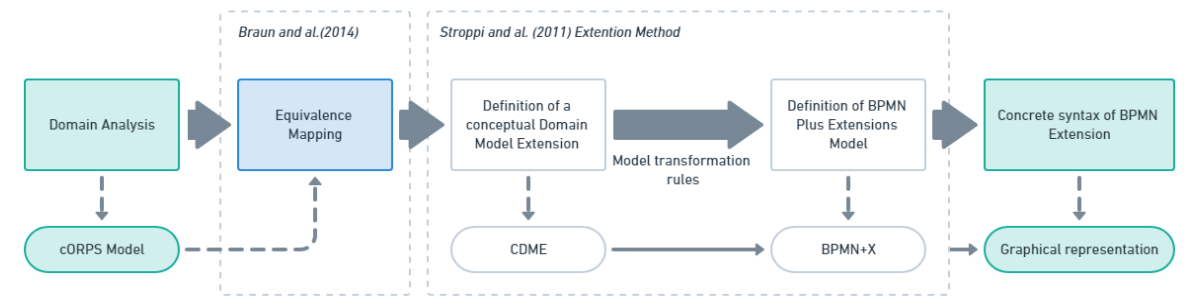

Fig. 1. Process for the development of domain specific BPMN extension

BPMN Metamodeling Extension. Based on the model transformation rules proposed by [31] we define an extension model $(\mathrm{BPMN}+\mathrm{X})$ by applying a set of transformation rules. The semantics and the abstract syntax of the extension model are based on BPMN extension mechanism. Depending on several rules in this phase [31], we had defined for each element, according to the domain specification, if we will use an existing BPMN element, define a new one or extend the attributes of the original BPMN elements; and how this changes will occur on BPMN meta-model. With the respect of the limited space of the paper, the entire transformation rules cannot be presented.

Proposed Graphical Notation. We proposed an advanced concrete syntax that defines the specific new graphical representation. As defined by the [27], the following extension can be made by: adding new markers or indicators, coloring graphical elements or changing the line style of a graphical element. According to these rules, Task elements are specified by colored borders and new markers that vary depending on the activity type. A pedagogical resource is represented as Data Object with a marker that reflects the selected Resource Type of the element. Subprocess line shape was also changed in order to differentiate it from generic Activity Element. The concrete graphical notation of the extension is presented in section 5.2.

\section{$5 \quad$ MOOC Authoring Tool: Elements and Architecture}

As described in section 3, BPMN represent a good alternative to conceive and deploy pedagogical scenarios, nevertheless it need of several adjustments to fulfill our conceptual needs. These adjustments can occur at three levels, namely: (1) the definition of pedagogical components based on cMOOC pedagogical principles. (2) The proposition of an authoring component for the creation of cMOOC learning procedures. (3) The development an automatic or semi-automatic mechanism for mapping learning procedures onto the online learning infrastructure (existing LMS). The first level corresponds to the domain analysis phase mentioned in the section 3. As a result, we had 
proposed a cMOOC Pedagogical Scenario Model, named cORPS [4]. It allows expressing the structural properties specific to a connectivist environment, as well as the temporal properties. To describe a cMOOC, the pedagogical scenario is based on a semantic description of the course. It consists of a description of the activities and resources it contains, but also of the properties of these entities, as well as their organization. The role of the pedagogical scenario as we perceive it is not merely to describe the actions that the learner must perform to complete a task. The teachers express the organization of pedagogical activities and their sequencing, bearing in mind that participants will participate in these learning activities in a non-linear context, in a conditional way [22, 30]. When the teachers create a pedagogical scenario, they have explicit access to these concepts, by creating a pedagogical workflow and then defining a temporal sequence of the proposed activities. They can also define different execution paths within the pedagogical scenario. In fact, activities are not necessarily organized in a sequential way. We have defined the root element by the scenario class, which is the entity that aggregates the different components of the pedagogical scenario. It is composed of one or several Learning Unit often with a fixed duration, usually equal to a week and can be composed of one or multiple lessons, which structure learning and assessment activities. A given activity can be assigned to one of the four categories: Aggregation (consultation and cognition), Remixing (communication, sharing and metacognition), Repurposing (production and collaboration) and Feed Forwarding (production sharing). We propose to add a category to the four categories corresponding to Evaluation activities (referring to evaluation activities: e-evaluation, peer evaluation or self-evaluation). Once the model scenario was defined, the aim was to embed it in a tool to design cMOOC-oriented scenarios. It is the main objective of the steps 2 and 3 cited above and described in the remainder of this section.

\section{1 cMOOC Authoring Tool Architecture}

In Section 3, we discussed the advantages of BPMN as a pedagogical modeling language. We should point out that this language has also been used to design various pedagogical situations in several contexts (face-to-face, hybrid, collaborative, etc.) [10, 27]. However, as explained in section 3 , in order to meet our requirements to provide the teacher with support for designing cMOOC-oriented pedagogical scenarios, the use of BPMN is not considered as it stands. The objective is not to rebuild a new platform, but to start from an existing tool and extend it. We therefore selected the BPMN.io tool, which is an open source web application that uses BPMN 2.0. The architecture of the BPMN.io application is composed of three main modules, as follows: Bpmn-js is the principal module of the tool, and controls the simple and visual human-computer interface used for creating, visualizing and validating BPMN schemas. This module displays and operates the toolbox elements, the modeling rules specific to BPMN 2.0, and the main modeling interface. It provides a viewer element for visualizing BPMN diagrams, and a modeler module to create, to edit and validate BPMN workflows. In this module, we have incorporated changes relating to the extension of the BPM notation and redefined the behavior of each element toolbox elements via the embedded business model expressed as a set of rules that regulates the behavior of each element. Bpmn-moddle 
embeds the metamodel defined by the BPMN 2.0 standard, and allows for mapping between the graphical notation and the elements of the BPMN metamodel. This module provides the appropriate modeling rules to validate BPMN workflows, and also allows reading and writing of XML files according to BPMN 2.0. In this module, we have added the elements of the our model through an extension of the BPMN metamodel. We have also modified the moddle-XML file to allow the identification of objects added to the toolbox (new elements specific to the building of cMOOC scenarios) and to indicate how these will be represented in the BPMN workflow. Finally, Diagram-js provides features that display and memorize changes in BPMN workflows during the conception process. In this module, we define the graphical aspect of the new notation. We add the MOOCAT ElementFactory module, which describes the visual appearance of each new element added to the toolbox and allows a mapping between the graphical representation and bpmn-moddle.

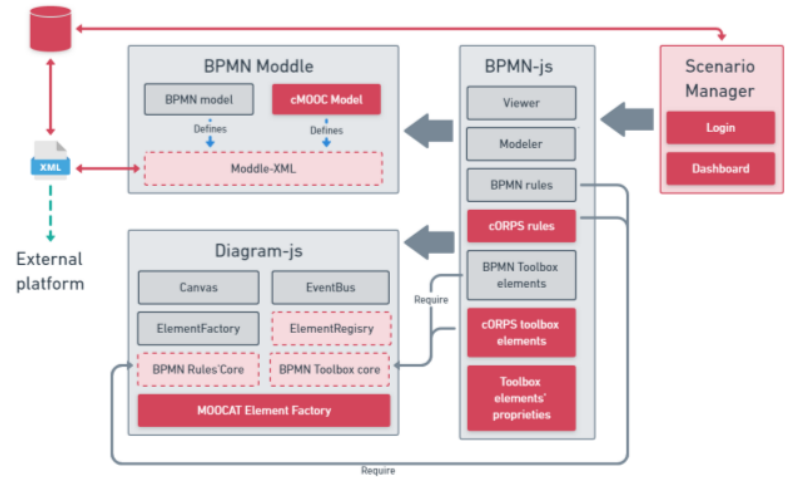

Fig. 2. Authoring Tool architecture

\subsection{MOOCAT: Features and Interfaces}

MOOCAT is a web application accessible via a web browser that not requires any prior installation. Once the teachers are connected to MOOCAT, they can either create a new scenario or modify an existing one (Fig.3, B). In the following, we consider that the teacher choose to create a new scenario (Fig. 3, B). After specifying the name of their scenario and choosing the blank model, the teacher is redirected to the conception page (Fig. 3, C). When starting a scenario conception with MOOCAT, the teachers start by creating a learning session. They had access to it via the toolbox (Fig.3. F) on the left under the "Learning session" block. In order to support the teachers, we propose to ensure that the modeling space (Fig3. D) is not empty when creating a new scenario. A first learning session is thus created by default. Commonly in MOOCs, one session represents one week. The teachers are then provided with an interface containing a pool, which can be renamed or deleted. They can then use the "Properties" section (Fig3. E) to specify the duration of this session (start date and end date). After creating there first session and specifying roles, the teachers can start creating there different lessons. We assume that a lesson is an entity that encompasses a number of activities. The teachers can thus continue his modeling by dragging from the toolbox the activities they want 
to model. In order to facilitate the identification of activities according to the four principles of a connectivist course, we have classified them into four blocks with different color codes. The different types of activities in the toolbox have been explicated in section 2. Each of the activities has its own properties. For example, for a consultation activity, the teachers specify whether it is a resource or an HTML page describing the activities to be carried out or presenting a description of the activity's progress. If it is a resource, they specify its type and the link to access it. Once the scenario modeling is complete, the teachers can possibly save the scenario in different formats (Fig3. D) or deploy it on an online platform ("Export to..." button). This action activates the transformation of the BPMN file into a file that can be imported by the learning platform.

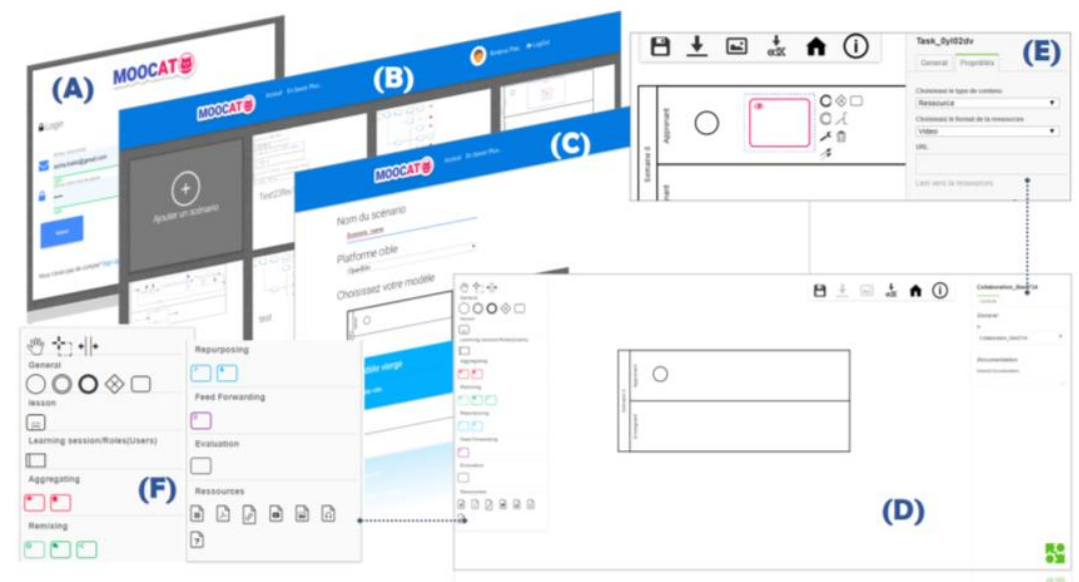

Fig. 3. Interfaces and features of proposed tool

\section{MOOCAT Scenario Operationalization Service}

In order to support the teacher, a service allowing the deployment of pedagogical scenarios carried out by MOOCAT has emerged. Operationalization represents an intermediate phase between learning and scenario design. It aims essentially to ensure that the scenario described by the teacher can be used and manipulated on a learning device while preserving the described pedagogical semantics [1]. In the literature, there are two types of approaches to operationalize pedagogical scenarios: a manual approach and an automated approach. [1] has classified these operationalization approaches into four categories: (1) Approaches based on the use of standards such as IMS-LD; (2) Approaches based on teachers' needs and practices [17, 32]; (3) Proprietary approaches proposed by platforms such as LAMS [15]; (4) Hybrid approaches based on processes and tools inspired and/or applied by model-driven engineering [11]. Our contribution is based on the last one. Consequently, we have implemented an operationalization service that allows teachers to automatically deploy their pedagogical scenarios on a specific target platform. In line with our work, we have provided a solution that allows transforming the pedagogical workflow into a deployable scenario. In order to demonstrate the technical feasibility of our proposal, we have chosen to develop importation 
modules for OpenEDX and moodle platforms. We thus proposed the approach that goes through two phases: (1) Transformation - Pretreatment. The aim is to propose a confrontation between the two models, in order to resolve all ambiguities and to match each concept in the MOOCAT scenario with a concept in the chosen platforms. It is a surjective transformation, i.e. each MOOCAT element has at least one correspondence on the platform. The general idea of the transformation algorithm is to: (i) Generate the BPMN pedagogical workflow. (ii) Create the tree structure of files from the information specified in the BPMN file. (iii) Transform the scenario into the format required by OpenEDX or Moodle. (2) Deployment. The operationalization module acts as a communication gateway from our tool to a learning platform. In this phase, the service automatically connects to the platform and retrieves the list of available courses. The teacher can choose a course from existing ones or create a new course. Finally, the deployment process is automatically executed, using the platform import function.

An extension of the transformation and deployment module to other platforms remains possible, as long as the target-learning platform provides import/export functionality. Therefore, for a given platform, it is first necessary to go through the confrontation phase; the purpose is to find a correspondence between the elements of a MOOCAT scenario and the scenario model of the target platform. An illustration of the overall process can be found at the following link ${ }^{1}$.

\section{$7 \quad$ Evaluation}

Objective and description. In this research, the contributions were evaluated and tested as they were specified through simulations and user tests, in order to confront them with the real needs of the target users. The final evaluation was established as part of an experimentation with 40 participants to evaluate the benefits of the extended notation and tool. Our objective is to evaluate the usability of MOOCAT as a cMOOCoriented pedagogical authoring tool and the expressivity of the proposed notation. In other words, we wanted to verify the ability of the proposed extension to express a cMOOC oriented pedagogical scenario. In order to reach the most diversified participants, a call for participation was broadcast to the TEL community through different mailing lists. We have also experimented our tool during a pedagogical scenario-building workshop with Master degree students that have previously designed pedagogical situations and manipulated different instructional design tools.

Experimental Protocol. The evaluation protocol we have adopted consists of three steps, namely: (1) Preparation. We provided participants with a MOOCAT user guide that explains the MOOCAT philosophy and describes the functionalities of the tool and an experimentation guide that describes the different steps to be performed during this evaluation as well as the scenario to be deployed. (2) Conception. This step aims to design a pedagogical scenario for a cMOOC course according to the instructions provided during the preparation phase. (3) Results. For this step, we provided participants with a questionnaire that they could complete at the end of the evaluation in order to

${ }^{1}$ https://youtu.be/JwRSyFxATUc 
validate the utility and usability of some aspects of MOOCAT and to obtain more information on the participants' experience.

Data collection. The methodology used to collect the data from this experimentation is based on two data sources, namely: (1) data derived directly from the work on MOOCAT, including produced scenarios; and (2) participant opinion data collected through questionnaires. The scenarios produced by the participants were analyzed using a rubric evaluation grid to assess, on a scale from one to three (1: low - 3: high). We examined all the scenarios and assigned a score for each criterion, then calculated the average scores, which were then compared to the median of two. At the end of the experimentation, participants were asked to complete an online questionnaire contained 25 closed-ended questions, evaluated using a 6-point Likert scale (from Strongly Disagree to Strongly Agree). The first part of the questionnaire focused mainly on the expressivity of the notation. The second part concerns the measurement of the usability of the tool, for this part we used the SUS questionnaire System Usability Scale [6].

Experimental results. All the scenarios created by the participants were collected and analyzed according to an evaluation grid that we defined. Table 1 presents the measured criteria and the average score for each criterion for all submitted scenarios. The criteria we have defined can be divided into two groups, qualifiable indicators that we have evaluated by observation (C2, C4 and C5), and quantifiable indicators that can be automatically calculated from the collected traces ( $\mathrm{C} 1$ and $\mathrm{C} 3$ ). Criteria $\mathrm{C} 2$ and $\mathrm{C} 4$ shows that participants were able to create structured and organized pedagogical scenarios. This shows that teachers can easily create pedagogical workflows, proving the advantages of using BPMN as a pedagogical scenario language. The averages obtained for criteria $\mathrm{C} 1$ and $\mathrm{C} 2$ show that the majority of participants were able to create a wellstructured cMOOC scenario that contained all the essential connectivist elements.

Table 1. Average scores per evaluation criterion for all submitted pedagogical scenarios

\begin{tabular}{l|lc}
$\mathbf{n}^{\circ}$ & Criterion & score/3 \\
\hline C1 & Number of designed weeks & 2,7 \\
C2 & Expressiveness of the scenario representation & 2,7 \\
C3 & Use of all connectivist scenario concepts & 2,5 \\
C4 & Relevance of the proposed learning resources and activities related to the course topic. & 2,5 \\
C5 & Visual representation and organization of the scenario & 2,7 \\
\hline
\end{tabular}

The analysis of the first part of the questionnaire aimed to determine whether the tool allowed for the simple design of a connectivist course. This section also assessed whether participants were satisfied with the tool and whether the tool's notation was easy to understand. Finally, it aimed to evaluate the potential of MOOCAT for designing a cMOOC course. In the first part of the questionnaire, 31 participants stated that the organization of the toolbox allowed them to identify the elements. In addition, 33 of the participants stated that the four connectivist activity blocks helped them to identify the activities and their usefulness. A total of 37 participants indicated that MOOCAT offered all the concepts required designing a cMOOC course, whereas only 3 of them did not agree with this statement. In addition, 34 indicated that the visual representation of a scenario was expressive and facilitated the course design. In the second part, the SUS questionnaire was used to measure the usability of MOOCAT. 
SUS is a popular and effective tool for assessing the usability of various systems [6]. It uses closed-ended questions with a Likert scale, which provides a 5-point gradation for each question ranging from"(1) totally disagree" to"(5) totally agree". Before calculating the SUS score, we pre-processed the participants' responses to remove any errors. In order to detect these errors, we used the grid presented by [23], which consists in considering all responses where the participant provided a score greater than 3 for all negative statements as incorrect. Of the 40 responses received, 6 were withdrawn. Overall, the average SUS score of all participants was 69.25 with a SD of 14.96. This score corresponds to the 55th percentile according to the standardization of [29].
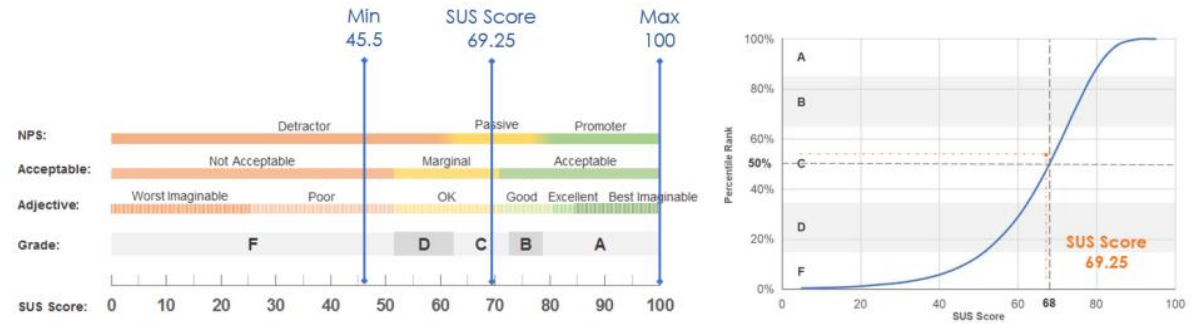

Fig. 4. SUS Score

In accordance with the empirical rule of interpretation of SUS scores [6], systems with scores under 50 are considered unacceptable, products with a score between 50 and 70 are marginally acceptable and those with a score above 70 are acceptable. By positioning the score obtained on the acceptability scale and the rating proposed by [6], the average SUS score of 69.25 indicates that MOOCAT is generally perceived as being close to the boundary between "marginally acceptable" and "acceptable" and between "OK" and "Good" for the notation.

\section{Conclusion}

The main objective of this research work is to support teachers in designing connectivist activities. We identify two steps leading from the design to the operationalization of a cMOOC-oriented scenario. The first consists of modeling the pedagogical scenario using a visual authoring tool; this editor is based on the BPMN graphical notation, and is aimed at teachers without specific technical knowledge or knowledge of the embedded model. We chose to adapt an existing open source BPMN modeling tool (BPMN. io) to embed our cMOOC scenario model. The second step consists of the automatic deployment of a scenario designed using MOOCAT on a MOOC platform. For this deployment phase, a web service solution was developed for the OpenEDX platforms. Our tool ensures that the mappings between the elements of its own scenario and those of the LMS (OpenEDX) are correct and comprehensible from both a semantic and a functionality point of view. These proposals were evaluated from utility and usability point of view. The findings confirm that MOOCAT can be used to design connectivist pedagogical scenarios and can provide all the necessary elements for the design of such courses. In our approach, the cMOOC is initially designed by the teacher, and learners are then encouraged to adapt the scenario according to their learning objectives. As a 
perspective of our work, we therefore consider that a methodology based on the codesign of a scenario that is currently in use would be a possible solution to this challenge, by giving access to the learners to MOOCAT with special and restricted roles and privileges. However, several scientific issues arise regarding the articulation of adaptation needs, the capitalization of these proposals, and the negotiation and validation of any changes carried out, especially in a massive environment.

\section{References}

1. Abedmouleh A, Laforcade P, Oubahssi L, Choquet C (2011) Operationalization of learning scenarios on existent Learning Management Systems the moodle case-study. ICSOFT 2011 - Proc 6th Int Conf Softw Database Technol 2:143-148. doi: 10.5220/0003486001430148

2. Adesina A, Molloy D (2010) Capturing and monitoring of learning process through a business process management (BPM) framework. In: Proc. of 3rd International Symposium for Engineering Education

3. Alario-Hoyos C, Pérez-Sanagustín M, Cormier D, Delgado-Kloos C (2014) Proposal for a Conceptual Framework for Educators to Describe andDesign MOOCs. J Univers Comput Sci 20:6-23. doi: 10.3217/jucs-020-01-0006

4. Bakki A, Oubahssi L, George S, Cherkaoui C (2017) A Model to Assist Pedagogical Scenario Building Process in cMOOCs. In: 2017 IEEE 17th International Conference on Advanced Learning Technologies (ICALT). pp 5-7

5. Bakki A, Oubahssi L, George S, Cherkaoui C (2019) MOOCAT: A visual authoring tool in the cMOOC context. Educ Inf Technol 24:1185-1209. doi: 10.1007/s10639-018-9807-2

6. Bangor A, Kortum P, Miller J (2009) Determining what individual SUS scores mean: Adding an adjective rating scale. J usability Stud 4:114-123. doi: 66.39.39.113

7. Barchino R, Hilera JR, De-Marcos L, Gutiérrez JM, Otón S, Gutiérrez JA, Martinez JJ, Jiménez L (2012) Interoperability between visual UML design applications and authoring tools for learning design. Inf Control Int J Innov Comput 8:845-865

8. Botturi L, Derntl M, Boot E, Figl K (2006) A classification framework for educational modeling languages in instructional design. In: 6th IEEE International Conference on Advanced Learning Technologies (ICALT 2006)

9. Braun R, Schlieter H, Burwitz M, Esswein W (2014) BPMN4CP: Design and implementation of a BPMN extension for clinical pathways. In: 2014 IEEE international conference on bioinformatics and biomedicine (BIBM). pp 9-16

10. Britain S (2004) A review of learning design: concept, specifications and tools. A Rep JISC E-learning Pedagog Program 2006

11. Caron P-A (2007) Bricoles : une approche dispositive des applications Web 2.0 utilisables pour enseigner. In: Actes de la conférence EIAH 2007. pp 137-142

12. Clow D (2013) MOOCs and the funnel of participation. In: Proceedings of the Third International Conference on Learning Analytics and Knowledge. pp 185-189

13. Consortium IMSGL, others (2003) IMS learning design specification. Retrieved Febr 7:2009

14. Da Costa J (2014) BPMN 2.0 pour la modélisation et l'implémentation de dispositifs pédagogiques orientés processus. University of Geneva

15. Dalziel J (2011) Visualising Learning Design in LAMS: A Historical View. Teach English with Technol 11:19-34

16. Downes S (2008) Places to go: Connectivism \& connective knowledge. Innov J Online Educ 5:6 
17. Ferraris C, Martel C, Vignollet L (2008) LDL for collaborative activities. In: Handbook of visual languages for instructional design: Theories and practices. IGI Global, pp 224-251

18. Glance DG, Forsey M, Riley M (2013) The pedagogical foundations of massive open online courses. First Monday 18

19. Helic D (2006) Technology-supported management of collaborative learning processes. Int J Learn Chang 1:285-298

20. Katsamani M, Retalis S (2013) Orchestrating learning activities using the CADMOS learning design tool. Res Learn Technol 21:1-12

21. Kop R (2011) The Challenges to Connectivist Learning on Open Online Networks: Learning Experiences during a Massive Open Online Course. Int Rev Res Open Distance Learn 12:19-37. doi: 10.19173/IRRODL.V12I3.882

22. Kopp M, Lackner E (2014) Do Moocs Need a Special Instructional Design? EDULEARN14 Proc 7138-7147

23. Mclellan S, Muddimer A, Peres SC (2012) The Effect of Experience on System Usability Scale Ratings. J Usability Stud 7:56-67

24. Morrison D (2013) The ultimate student guide to xMOOCs and CMOOCs. MOOC News Rev

25. Nodenot T (2007) Scénarisation pédagogique et modèles conceptuels d'un EIAH : Que peuvent apporter les langages visuels? Rev Int des Technol en Pédagogie Univ (RITPU)/International J Technol High Educ 4:85-102

26. O'Brien KL, Forte M, Mackey TP, Jacobson TE (2017) Metaliteracy as Pedagogical Framework for Learner-Centered Design in Three MOOC Platforms: Connectivist, Coursera and Canvas. Open Prax 9:267. doi: 10.5944/openpraxis.9.3.553

27. OMG (2011) Business Process Model and Notation. 95:206-242. doi: 10.1007/978-3-64225160-3

28. Pettenati MC, Cigognini ME (2007) Social Networking Theories and Tools to Support Connectivist Learning Activities. Int J Web-Based Learn Teach Technol 2:42-60. doi: 10.4018/jwltt.2007070103

29. Sauro J, Lewis JR (2011) When designing usability questionnaires, does it hurt to be positive? In: Proceedings of the SIGCHI Conference on Human Factors in Computing Systems. pp 2215-2224

30. Sergis S, Sampson DG, Pelliccione L (2016) Educational design for moocs: Design considerations for technology-supported learning at large scale. Lect Notes Educ Technol 39-71. doi: 10.1007/978-3-662-52925-6_3

31. Stroppi LJR, Chiotti O, Villarreal PD (2011) Extending BPMN 2.0: method and tool support. In: International Workshop on Business Process Modeling Notation. pp 59-73

32. Stylianakis G, Moumoutzis N, Arapi P, Mylonakis M, Christodoulakis S (2015) COLearn and open discovery space portal alignment: A case of enriching open learning infrastructures with collaborative learning capabilities. Proc 2014 Int Conf Interact Mob Commun Technol Learn IMCL 2014 252-256. doi: 10.1109/IMCTL.2014.7011142

33. Toven-Lindsey B, Rhoads RA, Lozano JB (2015) Virtually unlimited classrooms: Pedagogical practices in massive open online courses. internet High Educ 24:1-12 